\title{
Segregation of In to dislocations in InGaN
}

Matthew K. Horton ${ }^{1, *}$, , Sneha Rhode ${ }^{2, *}$,Suman-Lata Sahonta ${ }^{2}$, Menno J. Kappers ${ }^{2}$, Sarah J. Haigh $^{3}$, Timothy J. Pennycook ${ }^{4,5}$, Colin J. Humphreys ${ }^{2}$, Rajiv O. Dusane ${ }^{6}$, and Michelle A. Moram $^{l}$

1) Dept. Materials, Imperial College London, Exhibition Road, SW7 2AZ, UK.

2) Dept. Materials Science \& Metallurgy, University of Cambridge, Charles Babbage Road, Cambridge, CB3 OFS, UK.

3) School of Materials, University of Manchester, Manchester, M13 9PL.

4) SuperSTEM, STFC Daresbury Laboratories, Warrington, WA4 4AD, UK.

5) Department of Materials, University of Oxford, Parks Road, Oxford OX1 3PH, UK

6) Dept. Metallurgical Engineering and Materials Science, Indian Institute of Technology Bombay, Mumbai, 400076, India.

\section{ABSTRACT}

Dislocations are one-dimensional topological defects which occur frequently in functional thin film materials and which are known to degrade the performance of $\operatorname{In}_{\mathrm{x}} \mathrm{Ga}_{1-\mathrm{x}} \mathrm{N}$-based optoelectronic devices. Here, we show that large local deviations in alloy composition and atomic structure re expected to occur in and around dislocation cores in $\operatorname{In}_{x} \mathrm{Ga}_{1-x} \mathrm{~N}$ alloy thin 
films. We present energy-dispersive X-ray spectroscopy data supporting this result. The methods presented here are also widely applicable for predicting composition fluctuations associated with strain fields in other inorganic functional material thin films.

\section{KEYWORDS}

Dislocations, III-nitrides, Monte Carlo, alloy segregation, atomistic modeling, STEM-EDX

\section{MAIN TEXT}

Dislocations are ubiquitous one-dimensional topological defects that are found within thin films of nitride semiconductors, originating at the interface with the substrate, and threading up through the active region of the device before terminating at the crystal surface ${ }^{1}$. These dislocations can severely degrade device efficiencies ${ }^{2}$, and lifetimes ${ }^{3}$ and are responsible for a broad range of undesirable behavior such as leakage currents ${ }^{4}$ and properties such as reduced internal quantum efficiencies ${ }^{5}$ and defect states ${ }^{6,7,8,9,10}$ that can act as non-radiative recombination centers. $\operatorname{In}_{x} \mathrm{Ga}_{1-x} \mathrm{~N}$-based alloy semiconductors are used in light-emitting diodes ${ }^{11}$, laser diodes ${ }^{12}$ and solar cells ${ }^{13}$, which can be tuned to emit or absorb respectively over the entire visible spectrum by varying the $\mathrm{In}$ composition ${ }^{14} \cdot \operatorname{In}_{x} \mathrm{Ga}_{1-x} \mathrm{~N}$ is subject to very high threading dislocation densities of up to $10^{11} \mathrm{~cm}^{-2}$ and typically around $10^{9} \mathrm{~cm}^{-2}$ when grown by metalorganic vapourphase epitaxy ${ }^{15}$ (MOVPE), of which the majority have $a$-type ('edge') or $(a+c)$-type ('mixed') Burgers vectors with $<1 \%{ }^{16}$ being $c$-type ('screw'). High dislocation densities are associated with short lifetimes in InGaN-based optoelectronic devices ${ }^{17}$. The electronic properties of dislocations are determined by the local bonding in the region of the dislocation core ${ }^{8}$. It is therefore important to determine whether or not there are local differences in the alloy composition near dislocation cores in $\operatorname{In}_{x} \mathrm{Ga}_{1-\mathrm{x}} \mathrm{N}$. Such composition fluctuations are likely to 
affect the electronic properties of the dislocations and would therefore affect device performance.

Each dislocation is associated with a strain field determined by its Burgers vector. Since the In atom is larger than the host Ga atom, it is expected that if the In atoms are sufficiently mobile during growth, then they will segregate to the tensile part of the dislocation strain field ${ }^{18}$. Previous theoretical work has shown that the extreme case of a pure $\mathrm{InN} c$-type dislocation core in an $\operatorname{In}_{x} \mathrm{Ga}_{1-x} \mathrm{~N}$ alloy is more energetically favorable compared to the equivalent $\operatorname{In}_{x} \mathrm{Ga}_{1-x} \mathrm{~N}$ core ${ }^{19}$, and also that it is favorable for In atoms to bind to a $c$-type dislocation core in $\mathrm{GaN}^{20}$. Due to the sensitivity required to detect small variations in alloy concentration on short length scales, it is difficult to find unambiguous evidence for segregation. Early reports ${ }^{21}$ suggested In segregation to dislocations in InGaN but subsequent work indicated that apparent In segregation was actually an artifact produced by electron-beam induced sample damage in $\mathrm{TEM}^{22}$ which could occur preferentially at dislocation cores. Features have been observed in $3 \mathrm{D}$ atom probe data that could be explained by In segregation around dislocations ${ }^{23}$, and a combined cathodoluminescence and atomic force microscopy study by Sugahara et $a l .{ }^{24}$ has provided evidence supporting the segregation of In to $c$-type and $(a+c)$-type dislocations.

In this work, we simulate plausible equilibrium microstructures in $\operatorname{In}_{x} \mathrm{Ga}_{1-x} \mathrm{~N}$ for all Burgers vectors by applying a combination of classical atomistic simulations and Metropolis Monte Carlo methods. Empirical Stillinger-Weber-style potentials have been developed for $\mathrm{GaN}^{25,26}$ and $\mathrm{InN}^{27}$. These potentials have previously been used with success to study dislocations in $\mathrm{GaN}^{26,28,29} \mathrm{InN}^{30}$ and $\operatorname{In}_{x} \mathrm{Ga}_{1-x} \mathrm{~N}$ alloys ${ }^{31}$ including quantum dot systems ${ }^{32}$ and strained $\operatorname{In}_{x} \mathrm{Ga}_{1-x} \mathrm{~N}$ quantum well systems $^{33}$ where the potentials were additionally validated by comparison to $a b$ initio Density Functional Theory results. Here, we use these Stillinger-Weber potentials with the LAMMPS 
molecular dynamics code ${ }^{34}$ to implement our model. Our simulation cells are $64 a \times 64 \times 64 c$ unit cells in size, and contain a dislocation dipole defined initially by an appropriate displacement field $^{35}$. There are sometimes multiple atomic configurations possible at the core for each Burgers vector, and these are typically referred to by the number of atoms in the atomic rings that make up the core. For $a$-type dislocations, these are the 5-atom/7-atom ring, 8-atom ring and 4-atom ring cores $^{36}$. For $(a+c)$-type dislocations, double-5-atom/6-atom ring cores, 5-atom/7-atom ring $\operatorname{cores}^{28}$ and 9-atom/4-atom/7-atom ring cores $^{37}$ are possible. In this work, the 5/7-atom ring $a$ type core is favored since it is considered to be the most stable ${ }^{9}$, though other core types are considered, while the 5/7-atom ring $(a+c)$-type core is not studied since it has not been observed experimentally ${ }^{16}$ and instead the $9 / 4 / 7$-atom ring $(a+c)$-type core is favoured. Likewise, only the most stable $c$-type core ${ }^{38}$, and the double-5/6-atom ring a+c-type core ${ }^{28}$ were studied.

The initial structure is relaxed, and Ga atoms are replaced by In atoms at random until the desired composition is reached, before the cell is relaxed again. The cell dimensions are constrained such that they match that of an InGaN epilayer strained to a GaN substrate, such that our results are applicable to an $\operatorname{In}_{x} \mathrm{Ga}_{1-x} \mathrm{~N}$ quantum well in a GaN matrix, which would be typical for an $\mathrm{In}_{x} \mathrm{Ga}_{1-x} \mathrm{~N}$-based device.

To produce a plausible equilibrium microstructure from this initial random configuration, a Metropolis Monte Carlo algorithm ${ }^{39}$ is implemented under the canonical ensemble. First, the cell is relaxed to find the initial energy, $\mathrm{E}_{\text {initial }}$. Then an $\mathrm{In}$ and $\mathrm{Ga}$ atom are selected at random and swapped, and the cell relaxed again to find a trial energy, $\mathrm{E}_{\text {trial }}$. This trial swap is accepted with probability:

$$
\left(1, \quad\left(-\left(E_{\text {trial }}-E_{\text {initial }}\right) / k T\right)\right)
$$


for temperature $T$ and Boltzmann's constant $k$, or else the In and Ga atoms are swapped back to their original sites.

This is then repeated many times, with a single Monte Carlo step defined as $N$ attempted atom swaps, where $N$ is the number of In and Ga atoms in our model. This model implicitly assumes that atoms are not added or removed from the system, and that therefore the dislocations are not able to climb or dissociate during the simulation. This model can only predict equilibrium structures and is agnostic to diffusion pathways and how the atom swaps occur in practice. Despite this, it is likely that there is substantial mobility of alloy atoms on the growth surface during growth, with the activation energy for diffusion of In atoms being particularly low ${ }^{40}$, which would allow such rearrangement to take place. It is also possible that dislocations provide preferential diffusion pathways for In atoms ${ }^{41}$, or that atoms could swap by diffusion of vacancies. Additionally, previous kinetic Monte Carlo simulations for perfect, undefected $\mathrm{InGaN}^{42}$ incorporated vacancy diffusion explicitly, and found that cation vacancies in GaN do not interact with themselves or with In atoms, and that therefore if only the final, equilibrium microstructures are of interest, it is not necessary to employ a kinetic Monte Carlo model. The static Monte Carlo approach employed here has also been previously used with success to model undefected InGaN ${ }^{43}$.

To minimize computational complexity, swaps were only permitted within a cut-off radius of $14 a$ (where $a$ is the lattice parameter of $\mathrm{GaN}$ ) of the dislocation core, since only the immediate core region is likely to determine electronic properties: beyond this radius the binding energy of the In atoms to the core will be sufficiently $\operatorname{low}^{20}$ that the $\mathrm{In}$ and $\mathrm{Ga}$ atoms in the $\operatorname{In}_{\mathrm{x}} \mathrm{Ga}_{1-\mathrm{x}} \mathrm{N}$ alloy will be randomly distributed on the metal atom sites. The lattice is allowed to relax within the 
larger radius of $12 a$ when a swap is performed to prevent edge effects for swaps that occur with an atom near the $10 a$ boundary.

Simulations were performed at two compositions, $x=0.0625$ and $x=0.1875$, to match the InGaN compositions found in typical devices ${ }^{44}$, and also to avoid the higher In content regime where spinodal decomposition may occur ${ }^{45}$ and the assumption of a random alloy in the bulk no longer holds. These compositions correspond to $3.22 \mathrm{eV}$ and $2.81 \mathrm{eV}^{46}$ respectively at $0 \mathrm{~K}$ for a strained InGaN layer, and actual device emission wavelengths will depend on the quantum well thickness. The temperature is set to match typical growth conditions at $750^{\circ} \mathrm{C}$

Convergence to equilibrium was found after approximately a single Monte Carlo step, though the rate of convergence does vary between core types with c-type cores and higher In concentration cores taking longer to converge, as illustrated in Figure 1(a). The distribution of $E_{\text {trial }}-E_{\text {initial }}$ values is symmetrical and centered close to zero at $-0.00115 \mathrm{eV}$, confirming suitable geometry optimization has occurred for each attempted swap. After equilibration, the total energy of the cell fluctuates about a constant value. This gives an estimate of the difference in energies for the random core compared to the segregated core, $\Delta \mathrm{E}_{\text {segregation }}$, and shows that segregated cores are in all cases more energetically favorable, with the largest stabilization occurring for the $(a+c)$-type and $a$-type cores, as summarized in Table 1. The stated energy differences will necessarily depend on the choice of cut-off radius, since additional strain relaxation could occur due to segregation at larger radii in the additional volume allowed to relax away from the core. This has been verified by repeating the simulations with increasing cut-off radii for the $a$-type core, where the local composition at the core was found to be insensitive to the cut-off radius provided the cut-off radius is at least $10 a$, validating our choice of cut-off radii for these simulations. Relative comparison of $\Delta \mathrm{E}_{\text {segregation }}$ between core-types is considered 
appropriate provided that the cut-off radius is fixed for all simulations, as it has been in this work. In the case of a single In atom in GaN, a binding energy can be defined which is the difference in energy between the In atom at an bulk-like site compared to a site close to the dislocation core. If this binding energy is greater than approximately $1-2 k T^{18}$, it is thought that segregation would not occur. Our calculations show that the limiting radius beyond which the binding energy would be too low is approximately $18 \AA$ for an $(a+c)$-type core, well within our cut-off radius. The temperature-dependence of the Metropolis algorithm used in this work should take this effect into account implicitly.

In all cases, significant enrichment at the core was seen. Figure 1(b) shows the local composition at the core region relative to the bulk composition, whereby an enrichment of about double the bulk concentration observed for $a$-type and $(a+c)$-type cores at lower indium concentrations, with a slightly lower enrichment around $a$-type cores, as displayed in Table 1 .

Additional simulations were performed using the same InGaN simulation cells, but this time without constraining the in-plane $a$ lattice parameter to match that of GaN. A further simulation was also performed at a slightly lower temperature of $700^{\circ} \mathrm{C}$. In both cases, no significant change in the resulting core structures or extent of In segregation was found. The results showed minor run-to-run differences in In content at the core, but these differences were deemed not to be statistically significant relative to the expected statistical spread of In contents at the core resulting from the Monte Carlo model.

The spatial arrangement of In atoms for example equilibrium configurations can be seen in Figure 2. The dislocations with an $a$-type component can exhibit an enriched region where the larger In atoms relieve tensile strain on one side of the dislocation, with a corresponding depleted region on the opposite side. For the corresponding simulations of $c$-type dislocations in InGaN, 
an In-enriched region is also observed near the core, but there is no corresponding In-depleted region. This is because $c$-type cores have only a deviatoric stress field near the core. Therefore, the average In content within a $1 \mathrm{~nm}$ radius of the core is the same as the background In content for dislocations with an $a$-type component, but higher than the background In content for the $c$ type dislocations, despite the fact that In segregation at the core clearly occurs for all core types. Therefore, experimental compositional mapping techniques with sub-nm spatial resolution are required to detect In segregation at $a$-type and $(a+c)$-type dislocations in InGaN.

Simulations of the 4-atom ring a-type dislocation core (not shown) reveal that glide of this core is possible in response to local changes in In concentration at the core, whereby the 4-atom ring glides into an 8-atom ring configuration and then another 4 -atom ring. This was not observed for the other core structures, and the extent of glide is sensitive to the initial random distribution of In atoms, with different initial structures resulting in differing degrees of glide. In contrast, the 5/7-atom ring $a$-type core configuration remains constant under segregation, as seen in Figure 3(a,b), as does the $c$-type core.

Simulations of the $(a+c)$-type cores reveal a much more disrupted core structure than the $a$ type cores, as seen in Figure 3(c,d). For both In contents, the local bonding at the $(a+c)$-type core is highly disordered, with higher In contents associated with increased disorder. In some simulations, the resulting core configuration of the $(a+c)$-type core is ambiguous, and cores that resemble both the double-5/6-atom ring and the 9/4/7-atom ring $(a+c)$-type cores have been observed. Previous work on GaN has shown ${ }^{37}$ that the dislocation line energies of the double-5/6atom ring and 9/4/7-atom ring cores are similar, and it is likely that the resulting core structures in InGaN will therefore be highly sensitive to the local In content, which will vary randomly along the dislocation line even in the case of the segregated dislocation core structure. 
This large degree of segregation is expected to result in markedly different material properties. In addition to the band gap being highly sensitive to In composition, it can also affect the localization of excitons leading to increased efficiencies ${ }^{47,48}$. A locally higher In concentration will lead to localization of hole states $^{49}$ with chains of $\mathrm{In}-\mathrm{N}-\mathrm{In}-\ldots$ atoms being particularly associated with this localization ${ }^{50,51,52,53}$. These simulations predict much longer $\mathrm{In}-\mathrm{N}-\mathrm{In}-\ldots$ chains present in the vicinity of the core than would be expected in a random alloy of similar composition $^{43}$. Indeed, for the $x=0.1875$ simulations, in most cases there are unbroken chains of In-N-In- .. atoms running along the dislocation line, while for $x=0.0625$ there are many short In-N-In-... chains present in the vicinity of the dislocation core.

To test these theoretical predictions, a representative $\operatorname{In}_{x} \mathrm{Ga}_{1-x} \mathrm{~N}$ epilayer of composition $x=$ $0.06 \pm 0.01^{54}$, was grown on low-dislocation density GaN templates grown on $c$-plane sapphire by MOVPE at temperatures typically used for growth of InGaN-based device structures (710$750^{\circ} \mathrm{C}$ ), and with growth parameters optimized as given in previous studies ${ }^{55}$.

This was then studied using energy-dispersive X-ray spectroscopy (EDXS) and high resolution high-angle annular dark field (HAADF) imaging on an aberration-corrected Titan G2 ChemiSTEM electron microscope. The $(a+c)$-type cores were studied because they are (a) easier to locate than $a$-type cores, (b) present in our samples in proportions (in contrast to the pure $c$ type cores which comprise only $\sim 1 \%$ of all dislocations in our samples), (c) they reveal the effects of both $a$-type and $c$-type Burgers vectors components and (d) the highest energetic stabilization of In segregation is predicted for this core type and therefore it is most likely to be observed experimentally.

Figure 4 shows an $(a+c)$-type dislocation core, as identified by Burgers circuit analysis that reveals the $a$-type component, while the presence of Eshelby-Stroh twist ${ }^{56}$ confirms the c-type 
component. A V-pit marks the termination of the dislocation core at the film surface, as is typical for $\operatorname{In}_{x} \mathrm{Ga}_{1-x} \mathrm{~N}$ epilayers ${ }^{57}$, with a side length of approximately $40 \mathrm{~nm}$. Precise identification of the core configuration is not possible due to ambiguous contrast at the core, which could be consistent with a disrupted, rather than highly symmetrical, core configuration consistent with the highly segregated In-rich cores predicted as seen in Figure 3(c). Local strain-relaxation at the $\mathrm{V}$-pit, in addition to the In atoms preferentially occupying sites of reduced $\mathrm{N}$ coordination as the In-N bond is longer and weaker than the Ga-N bond ${ }^{58,59}$, would mean that segregation of In to the V-pit edges would be expected, and this has been observed in our samples. However, this is not a sufficient explanation for the high-In concentration streak in Figure 4(c), since this is only seen in the direction of a single V-pit edge rather than all six edges. Geometric phase analysis ${ }^{60}$ of the STEM-HAADF micrograph of the core region confirms that the segregation is correlated approximately the region of tensile stress around the dislocation, as shown in Figure 4(d). The semi-quantitative absorption corrected Cliff-Lorimer ${ }^{61}$ analysis shown in Fig4(e) suggests an In composition of $\mathrm{x} \sim 0.04 \pm 0.01$ in the matrix (at a specimen thickness of $150 \mathrm{~nm}$ ). This value is less than that which is revealed by a combined X-ray diffraction and Rutherford back-scattering study on the same material ${ }^{54}$, but without standard specimens of known composition absolute quantification of EDXS data is challenging, especially for the four detector geometry of the Super-X detector system where the effects of sample tilt and channeling are still being investigated. Relative concentrations are more reliable and the line scan in Fig4(d) shows an In enrichment at the dislocation core of approximately double that found in the bulk, which is in good agreement with our theoretical predictions. Additionally, due to scale of the streak and the size of the STEM probe, channeling artifacts are unlikely to explain this strong In signal. However, the length of the streak, at approximately $14 \mathrm{~nm}$, is much longer than that predicted 
theoretically. A possible explanation is that movement of the dislocation during growth tracks an In-rich trail in its path, thus explaining the streak observed, or that there is a more complex interaction between the V-pit and the dislocation resulting in a larger energetic stabilization of segregation than would otherwise be expected.

In this work, we have shown that the local concentration of In surrounding dislocations is likely to be very different compared to the bulk. This has important consequences for future modeling of dislocation cores and their properties, since the assumption of a core structure that reflects that of the host material does not hold. Segregation of the degree predicted here could significantly affect electronic properties of dislocations and hence affect device performance. The method presented here has a wide arrange of applicability not just to other nitride systems, but other inorganic functional material thin films including oxides where properties of dislocations are of considerable interest ${ }^{62,63}$, and could be applied more generally to predict composition fluctuations for any strain field.

\begin{tabular}{|l|l|l|l|l|}
\cline { 2 - 5 } \multicolumn{2}{l|}{} & \multicolumn{2}{l|}{$\Delta \mathrm{E}_{\text {segregation }}\left[\mathrm{eV} \AA^{-1}\right]$} & \multicolumn{2}{l|}{$x_{\text {core }} / x$} \\
\hline Core Type & $x=0.0625$ & $x=0.1875$ & $x=0.0625$ & $x=0.1875$ \\
\hline$a$-type & $-1.40 \pm 0.11$ & $-2.47 \pm 0.10$ & $2.25 \pm 0.27$ & $1.34 \pm 0.07$ \\
\hline$c$-type & $-0.62 \pm 0.09$ & $-1.53 \pm 0.11$ & $2.34 \pm 0.24$ & $1.56 \pm 0.07$ \\
\hline$(a+c)$-type & $-1.97 \pm 0.12$ & $-3.03 \pm 0.10$ & $2.73 \pm 0.27$ & $1.71 \pm 0.10$ \\
\hline
\end{tabular}


Table 1. Summary of difference in total energies of the simulation cell per unit dislocation line length for the random and segregated $\operatorname{In}_{\mathrm{x}} \mathrm{Ga}_{1-\mathrm{x}} \mathrm{N}$ dislocation cores, and a summary of the average composition in the core region at equilibrium, $x_{\text {core }}$, relative to the bulk composition, $x$.
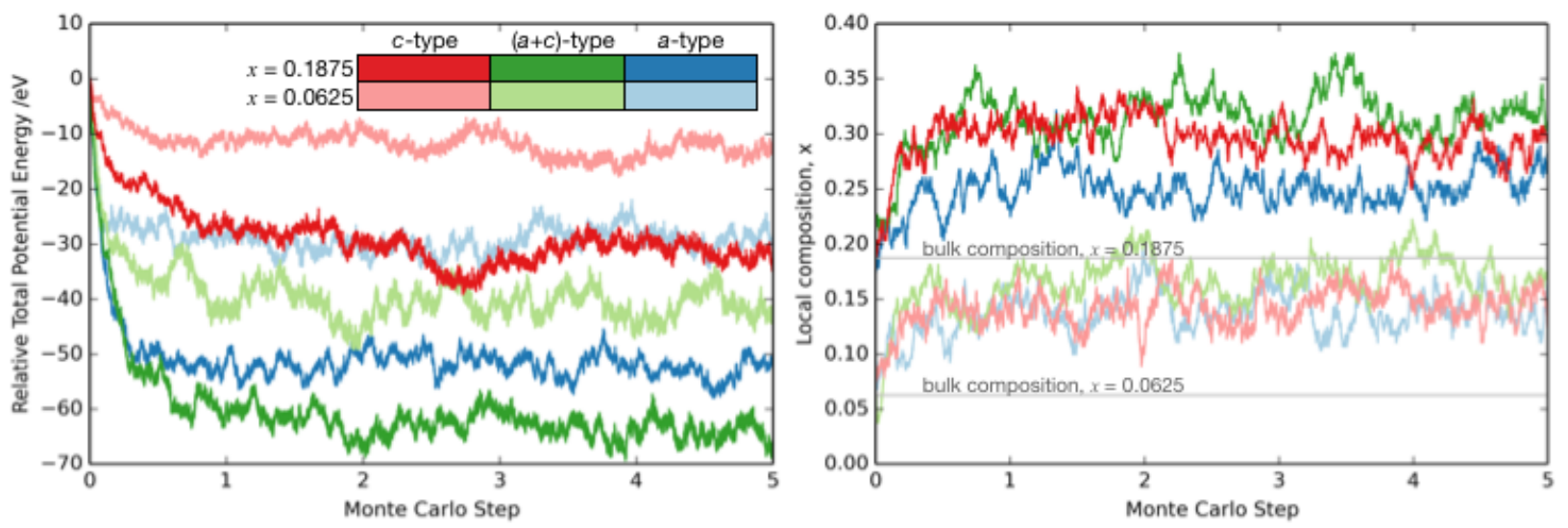

Figure 1. (a) Total potential energy of the system relative to initial random configuration, showing convergence to equilibrium. (b) Local composition at the dislocation core region, $x_{\text {core }}$, defined as a radius of $1 \mathrm{~nm}$ from the center of the dislocation, against Monte Carlo step. 

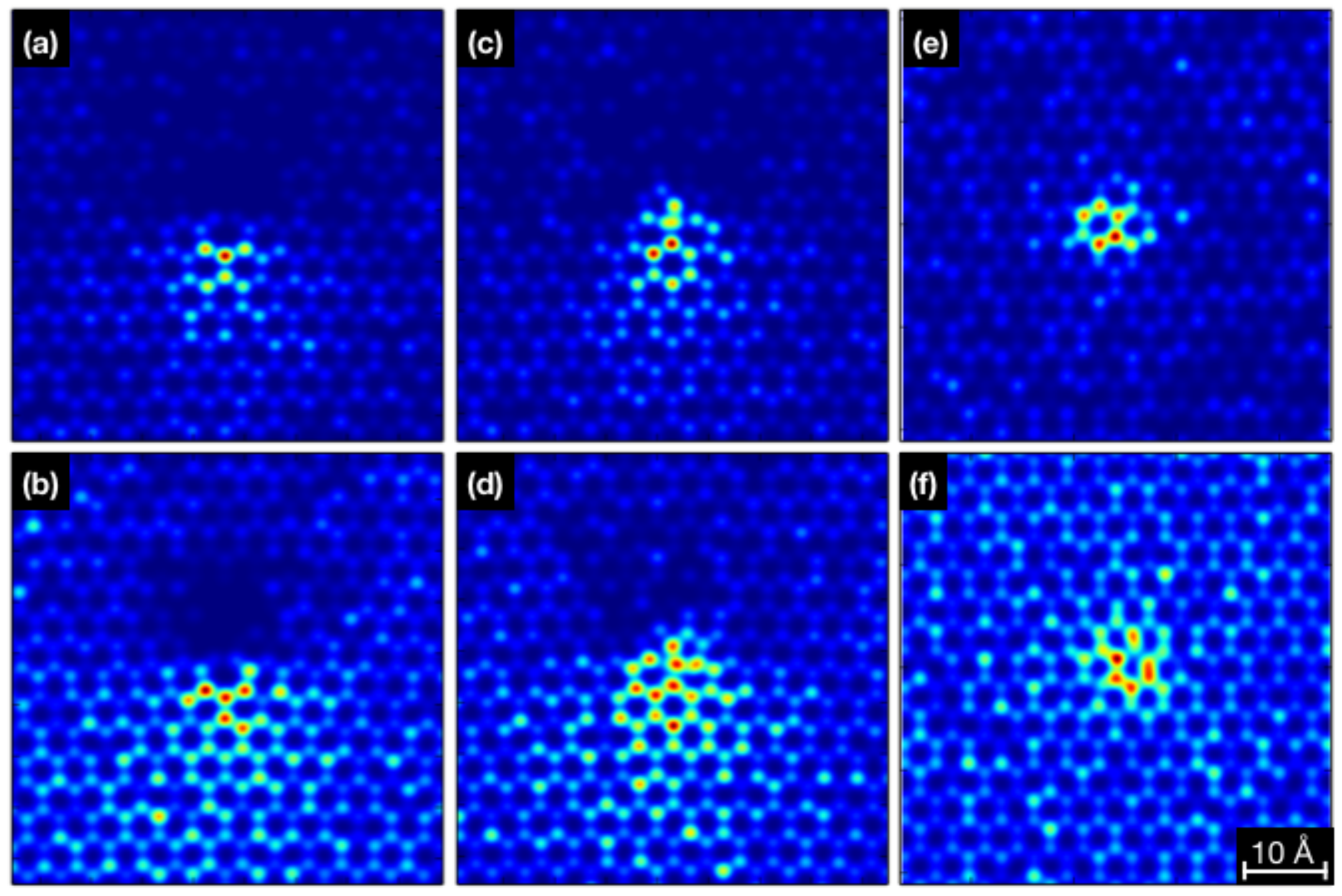

Figure 2. Kernel density estimators showing the distribution of indium atoms averaged from four different equilibrium configurations of $a$-type cores for compositions (a) $x=0.0625$ and (b) $x=0.1875,(\mathrm{a}+\mathrm{c})$-type cores for (c) $x=0.0625$ and (d) $x=0.1875$ and c-type cores for (e) $x=$ 0.0625 and (f) $x=0.1875$. Plots are projections along the [0001] direction, with axes given in angstroms and dislocation cores located at the center of their respective plots. Color scale is red to blue, with red giving a high probability of finding an indium atom at that location, and blue a low probability. 

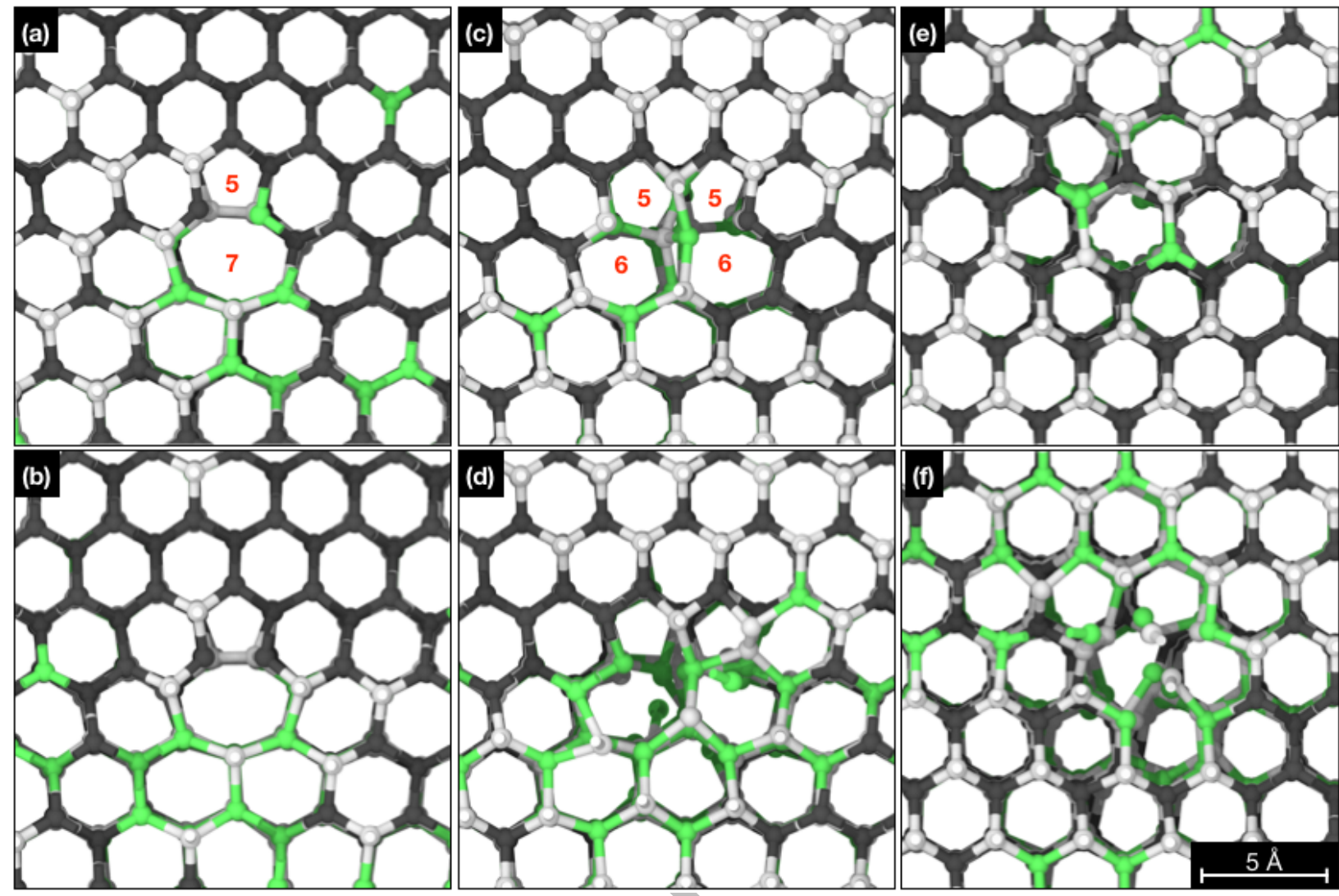

Figure 3. Example equilibrium core configurations for $a$-type cores for (a) $x=0.0625$ and (b) $x$ $=0.1875,(a+c)$-type cores for (c) $x=0.0625$ and (d) $x=0.1875$ and $c$-type cores for (e) $x=$ 0.0625 and (f) $x=0.1875$. The 5/7-atom ring $a$-type core structure and the double-5/6-atom ring $(a+c)$-type core structure are indicated by the numbers in red. $\mathrm{N}$ atoms shown in light grey, Ga atoms in dark grey, In atoms in green, rendered using Ovito ${ }^{64}$. 

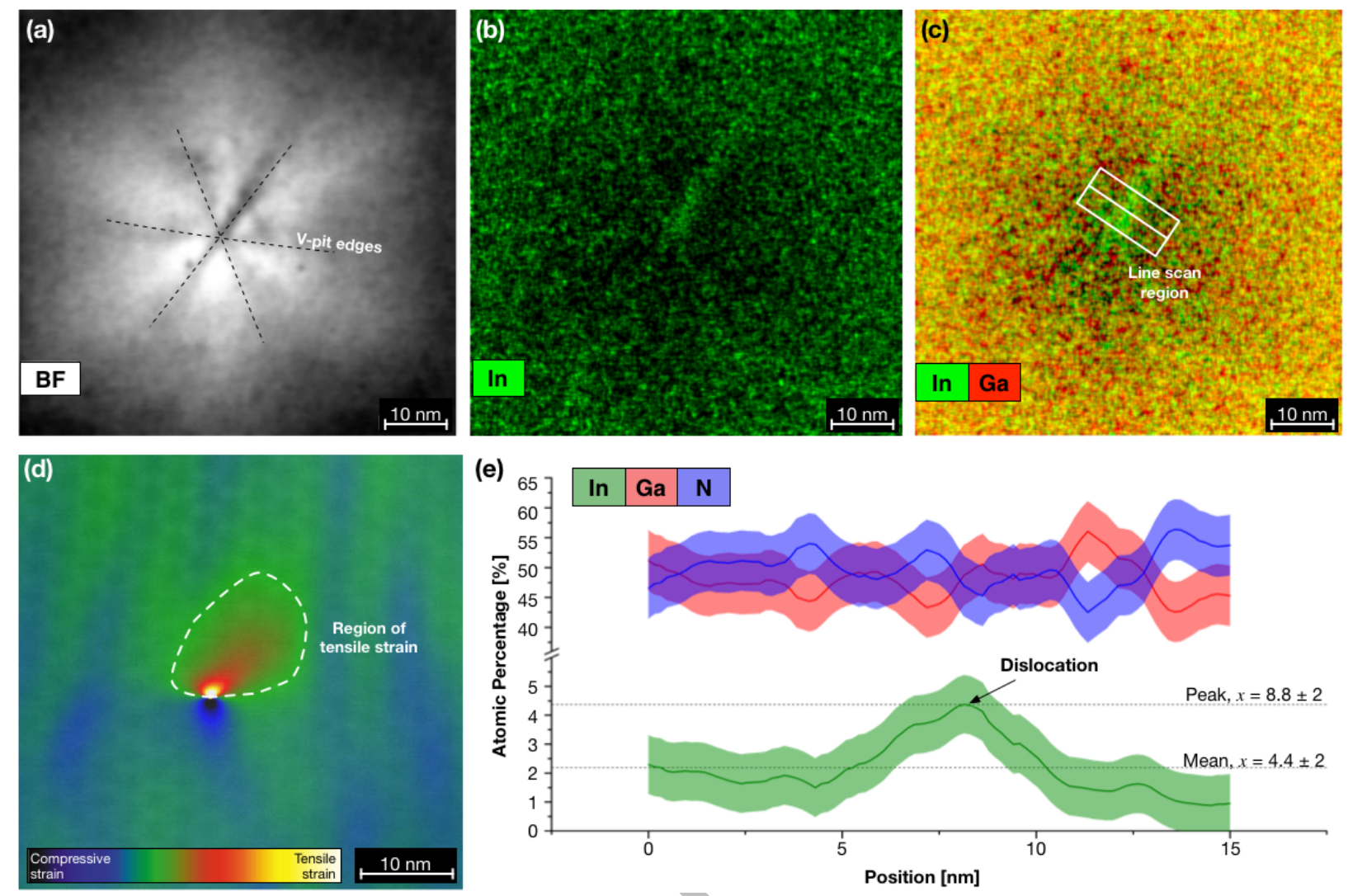

Figure 4. (a) Bright-field image of an $(a+c)$-type dislocation ending as a V-pit at the surface of our sample, with approximate location of V-pit edges marked, and its corresponding EDXS elemental map (b) showing the In signals and (c) In and Ga signals confirming the segregation of In at the apex of the V-pit and along the direction of a V-pit edge. (d) shows the geometric phase analysis of the dislocation core, showing the $\varepsilon_{\mathrm{xx}}$ strain component with characteristic compression and dilatation of the region close to the dislocation core. (e) is a line profile for the rectangle shown in (c), perpendicular to the In-rich streak, showing enrichment at the dislocation core, with peak composition at the core approximately double relative to the bulk, with shaded regions showing approximate error in quantification.

\section{AUTHOR INFORMATION}

\section{Corresponding Author}


*Matthew K. Horton is the corresponding author, contact via email: m.horton11@imperial.ac.uk

\section{Author Contributions}

The manuscript was written through contributions of all authors. All authors have given approval to the final version of the manuscript. $\ddagger$ Matthew K. Horton and Sneha K. Rhode have contributed equally.

\section{Funding Sources}

This work was funded in part by the Cambridge Commonwealth trust, St. John's College and the EPSRC. SKR is funded through the Cambridge-India Partnership Fund and Indian Institute of Technology Bombay via a scholarship. MAM acknowledges support from the Royal Society through a University Research Fellowship. Additional support was provided by the EPSRC through the UK National Facility for Aberration-Corrected STEM (SuperSTEM). The Titan 80$200 \mathrm{kV}$ ChemiSTEM ${ }^{\mathrm{TM}}$ was funded through HM Government (UK) and is associated with the capabilities of the University of Manchester Nuclear Manufacturing (NUMAN) capabilities. SJH acknowledges funding from the Defence Treat Reduction Agency (DTRA) USA (grant number HDTRA1-12-1-0013)

\section{ACKNOWLEDGMENT}

This work was funded in part by the Cambridge Commonwealth trust, St. John's College and the EPSRC. SKR is funded through the Cambridge-India Partnership Fund and Indian Institute of Technology Bombay via a scholarship. MAM acknowledges support from the Royal Society through a University Research Fellowship. Additional support was provided by the EPSRC through the UK National Facility for Aberration-Corrected STEM (SuperSTEM). The Titan 80$200 \mathrm{kV}$ ChemiSTEM ${ }^{\mathrm{TM}}$ was funded through HM Government (UK) and is associated with the 
capabilities of the University of Manchester Nuclear Manufacturing (NUMAN) capabilities. SJH acknowledges funding from the Defence Treat Reduction Agency (DTRA) USA (grant number HDTRA1-12-1-0013)

\section{ABBREVIATIONS}

MOVPE, metalorganic vapour-phase epitaxy; STEM, scanning transmission electron microscopy; HAADF, high-angle annular dark-field; EDXS, electron dispersive X-ray spectroscopy.

\section{REFERENCES}

(1) Bennett, S. E. Dislocations and their reduction in GaN. Mater Sci Tech-Lond 2010, 26, 1017-1028 DOI: 10.1179/026708310X12668415533685.

(2) Schubert, M. F.; Chhajed, S.; Kim, J. K.; Schubert, E. F.; Koleske, D. D.; Crawford, M. H.; Lee, S. R.; Fischer, A. J.; Thaler, G.; Banas, M. A. Effect of dislocation density on efficiency droop in GaInN/GaN light-emitting diodes. Applied Physics Letters 2007, 91, 231114 DOI: 10.1063/1.2822442.

(3) Schubert, M. F.; Xu, J.; Kim, J. K.; Schubert, E. F.; Kim, M. H.; Yoon, S.; Lee, S. M.; Sone, C.; Sakong, T.; Park, Y. Polarization-matched GaInNAlGaInN multi-quantum-well lightemitting diodes with reduced efficiency droop. Applied Physics Letters 2008, 93, 041102 DOI: $10.1063 / 1.2963029$.

(4) Hsu, J. W. P.; Manfra, M. J.; Molnar, R. J.; Heying, B.; Speck, J. S. Direct imaging of reverse-bias leakage through pure screw dislocations in GaN films grown by molecular beam epitaxy on GaN templates. Applied Physics Letters 2002, 81, 79 DOI: 10.1063/1.1490147. 
(5) Kneissl, M.; Kolbe, T.; Chua, C.; Kueller, V.; Lobo, N.; Stellmach, J.; Knauer, A.; Rodriguez, H.; Einfeldt, S.; Yang, Z.; et al. Advances in group III-nitride-based deep UV lightemitting diode technology. Semicond. Sci. Technol. 2011, 26, 014036 DOI: 10.1088/02681242/26/1/014036.

(6) Elsner, J.; Jones, R.; Sitch, P.; Porezag, V.; Elstner, M.; Frauenheim, T.; Heggie, M.; Öberg, S.; Briddon, P. Theory of Threading Edge and Screw Dislocations in GaN. Phys. Rev. Lett. 1997, 79, 3672-3675 DOI: 10.1103/PhysRevLett.79.3672.

(7) Blumenau, A. T.; Fall, C. J.; Elsner, J.; Jones, R.; Heggie, M. I.; Frauenheim, T. A theoretical investigation of dislocations in cubic and hexagonal gallium nitride. phys. stat. sol. (c) 2003, 0, 1684-1709 DOI: 10.1002/pssc.200303126.

(8) Fall, C.; Jones, R.; Briddon, P.; Blumenau, A.; Frauenheim, T.; Heggie, M. Influence of dislocations on electron energy-loss spectra in gallium nitride. Phys $\operatorname{Rev} B$ 2002, 65 DOI: 10.1103/PhysRevB.65.245304.

(9) Lymperakis, L.; Neugebauer, J.; Albrecht, M.; Remmele, T.; Strunk, H. Strain Induced Deep Electronic States around Threading Dislocations in GaN. Phys. Rev. Lett. 2004, 93 DOI: 10.1103/PhysRevLett.93.196401.

(10) Lee, S.; Belkhir, M.; Zhu, X.; Lee, Y.; Hwang, Y.; Frauenheim, T. Electronic structures of GaN edge dislocations. Phys Rev B 2000, 61, 16033-16039 DOI: 10.1103/PhysRevB.61.16033.

(11) Nakamura, S.; Mukai, T.; Senoh, M. High-Power GaN P-N Junction Blue-Light-Emitting Diodes. Jpn. J. Appl. Phys. 1991, 30, L1998-L2001 DOI: 10.1143/JJAP.30.L1998. 
(12) Nakamura, S. The Roles of Structural Imperfections in InGaN-Based Blue LightEmitting Diodes and Laser Diodes. Science 1998, 281, 956-961 DOI: 10.1126/science.281.5379.956.

(13) Jani, O.; Ferguson, I.; Honsberg, C.; Kurtz, S. Design and characterization of GaNMnaN solar cells. Applied Physics Letters 2007, 91, 132117 DOI: 10.1063/1.2793180.

(14) Mukai, T.; Yamada, M.; Nakamura, S. Characteristics of InGaN-Based UV/Blue/Green/Amber/Red Light-Emitting Diodes. Jpn. J. Appl. Phys. 1999, 38, 3976-3981 DOI: 10.1143/JJAP.38.3976.

(15) Moram, M. A.; Ghedia, C. S.; Rao, D. V. S.; Barnard, J. S.; Zhang, Y.; Kappers, M. J.; Humphreys, C. J. On the origin of threading dislocations in GaN films. J. Appl. Phys. 2009, 106, 073513 DOI: $10.1063 / 1.3225920$.

(16) Rhode, S. K.; Horton, M. K.; Kappers, M. J.; Zhang, S.; Humphreys, C. J.; Dusane, R. O.; Sahonta, S. L.; Moram, M. A. Mg Doping Affects Dislocation Core Structures in GaN. Phys. Rev. Lett. 2013, 111, 025502 DOI: 10.1103/PhysRevLett.111.025502.

(17) $\mathrm{Wu}, \mathrm{J}$. When group-III nitrides go infrared: New properties and perspectives. J. Appl. Phys. 2009, 106,011101 DOI: 10.1063/1.3155798.

(18) Fiore, N. F.; Bauer, C. L. Binding of solute atoms to dislocations. Progress in Materials Science 1968, 13, 85-134 DOI: 10.1016/0079-6425(68)90019-4.

(19) Lei, H.; Chen, J.; Ruterana, P. Influences of the biaxial strain and c-screw dislocation on the clustering in InGaN alloys. J. Appl. Phys. 2010, 108, 103503 DOI: 10.1063/1.3509147. 
(20) Lei, H.; Chen, J.; Ruterana, P. Role of c-screw dislocations on indium segregation in InGaN and InAlN alloys. Applied Physics Letters 2010, 96, 161901 DOI: 10.1063/1.3394007.

(21) Duxbury, N.; Bangert, U.; Dawson, P.; Thrush, E. J.; Van der Stricht, W.; Jacobs, K.; Moerman, I. Indium segregation in InGaN quantum-well structures. Applied Physics Letters 2000, 76, 1600 DOI: 10.1063/1.126108.

(22) Smeeton, T. M.; Humphreys, C. J.; Barnard, J. S.; Kappers, M. J. The impact of electron beam damage on the detection of indium-rich localisation centres in InGaN quantum wells using transmission electron microscopy. J Mater Sci 2006, 41, 2729-2737 DOI: 10.1007/s10853-0067876-x.

(23) Müller, M.; Smith, G. D. W.; Gault, B.; Grovenor, C. R. M. Phase separation in thick InGaN layers - A quantitative, nanoscale study by pulsed laser atom probe tomography. Acta Materialia 2012, 60, 4277-4285 DOI: 10.1016/j.actamat.2012.04.030.

(24) Sugahara, T.; Hao, M.; Wang, T.; Nakagawa, D.; Naoi, Y.; Nishino, K.; Sakai, S. Role of Dislocation in InGaN Phase Separation. Jpn. J. Appl. Phys. 1998, 37, L1195-L1198 DOI: 10.1143/JJAP.37.L1195.

(25) Aïchoune, N.; Potin, V.; Ruterana, P.; Hairie, A.; Nouet, G.; Paumier, E. An empirical potential for the calculation of the atomic structure of extended defects in wurtzite GaN. Computational Materials Science 2000, 17, 380-383 DOI: 10.1016/S0927-0256(00)00056-2.

(26) Béré, A.; Serra, A. On the atomic structures, mobility and interactions of extended defects in GaN: dislocations, tilt and twin boundaries. Philosophical Magazine 2006, 86, 21592192 DOI: 10.1080/14786430600640486. 
(27) Lei, H. P.; Chen, J.; Petit, S.; Ruterana, P.; Jiang, X. Y.; Nouet, G. Stillinger-Weber parameters for In and $\mathrm{N}$ atoms. Superlattice Microst 2006, 40, 464-469 DOI: 10.1016/j.spmi.2006.09.010.

(28) Belabbas, I.; Béré, A.; Chen, J.; Petit, S.; Belkhir, M.; Ruterana, P.; Nouet, G. Atomistic modeling of the $(\mathrm{a}+\mathrm{c})$-mixed dislocation core in wurtzite GaN. Phys Rev B 2007, 75, 115201 DOI: 10.1103/PhysRevB.75.115201.

(29) Béré, A.; Chen, J.; Ruterana, P.; Serra, A.; Nouet, G. The atomic configurations of the a threading dislocation in GaN. Computational Materials Science 2002, 24, 144-147 DOI: 10.1016/S0927-0256(02)00180-5.

(30) Lei, H. P.; Ruterana, P.; Nouet, G.; Jiang, X. Y.; Chen, J. Core structures of the a-edge dislocation in InN. Applied Physics Letters 2007, 90, 111901 DOI: 10.1063/1.2712799.

(31) Rosenauer, A.; Mehrtens, T.; Müller,K.; Gries, K.; Schowalter, M.; Satyam, P. V.; Bley, S.; Tessarek, C.; Hommel, D.; Sebald, K.; et al. Composition mapping in InGaN by scanning transmission electron microscopy. Ultramicroscopy 2011, 111, 1316-1327 DOI: 10.1016/j.ultramic.2011.04.009.

(32) Zhang, Z.; Chatterjee, A.; Grein, C.; Ciani, A. J.; Chung, P. W. Atomic-scale modeling of $\mathrm{In}_{\mathrm{x}} \mathrm{Ga}_{1-\mathrm{x}} \mathrm{N}$ quantum dot self-assembly. Journal of Vacuum Science \& Technology $B$ : Microelectronics and Nanometer Structures 2011, 29, 03C133 DOI: 10.1116/1.3579462.

(33) Lei, H.; Chen, J.; Jiang, X.; Nouet, G. Microstructure analysis in strained-InGaN/GaN multiple quantum wells. Microelectronics Journal 2009, 40, 342-345 DOI: 10.1016/j.mejo.2008.07.068. 
(34) Plimpton, S. Fast parallel algorithms for short-range molecular dynamics. Journal of Computational Physics 1995, 117, 1-19 DOI: 10.1006/jcph.1995.1039.

(35) Hirth, J. P.; Lothe, J. Theory of Dislocations; 2nd ed.; Krieger Publishing Company, 1991.

(36) Belabbas, I.; Ruterana, P.; Chen, J.; Nouet, G. The atomic and electronic structure of dislocations in Ga-based nitride semiconductors. Philosophical Magazine 2006, 86, 2241-2269 DOI: $10.1080 / 14786430600651996$.

(37) Horton, M. K.; Rhode, S. L.; Moram, M. A. Structure and electronic properties of mixed (a+c) dislocation cores in GaN. J. Appl. Phys. 2014, 116, 063710 DOI: 10.1063/1.4893030.

(38) Belabbas, I.; Chen, J.; Nouet, G. A new atomistic model for the threading screw dislocation core in wurtzite GaN. Computational Materials Science 2012, 51, 206-216 DOI: 10.1016/j.commatsci.2011.07.051.

(39) Frenkel, D.; Smit, B. Understanding molecular simulation: from algorithms to applications; Elsevier (formerly published by Academic Press), 2002; Vol. 1.

(40) Neugebauer, J.; Zywietz, T.; Scheffler, M.; Northrup, J.; Chen, H.; Feenstra, R. Adatom Kinetics On and Below the Surface: The Existence of a New Diffusion Channel. Phys. Rev. Lett. 2003,90,056101 DOI: 10.1103/PhysRevLett.90.056101.

(41) Love, G. Dislocation pipe diffusion. Acta Metallurgica 1964, 12, 731-737 DOI: 10.1016/0001-6160(64)90220-2. 
(42) Ganchenkova, M.; Borodin, V.; Laaksonen, K.; Nieminen, R. Modeling the compositional instability in wurtzite $\mathrm{Ga}_{1-\mathrm{x}} \operatorname{In}_{\mathrm{x}} \mathrm{N}$. Phys Rev $B$ 2008, 77, 075207 DOI: 10.1103/PhysRevB.77.075207.

(43) Chan, J. A.; Liu, J. Z.; Zunger, A. Bridging the gap between atomic microstructure and electronic properties of alloys: The case of (In,Ga)N. Phys Rev B 2010, 82, 045112 DOI: 10.1103/PhysRevB.82.045112.

(44) Morkoç, H. Handbook of Nitride Semiconductors and Devices: Materials Properties, Physics and Growth; Wiley: Weinheim, Germany, 2009; Vol. 1, pp. 1-129.

(45) Stringfellow, G. B. Microstructures produced during the epitaxial growth of InGaN alloys. J Cryst Growth 2010, 312, 735-749 DOI: 10.1016/j.jerysgro.2009.12.018.

(46) Sakalauskas, E.; Tuna, Ö.; Kraus, A.; Bremers, H.; Rossow, U.; Giesen, C.; Heuken, M.; Hangleiter, A.; Gobsch, G.; Goldhahn, R. Dielectric function and bowing parameters of InGaN alloys. physica status solidi (b) 2012,249, 485-488 DOI: 10.1002/pssb.201100334.

(47) Kachkanov, V.; O'Donnell, K. P.; Pereira, S.; Martin, R. W. Localization of excitation in InGaN epilayers. Philosophical Magazine 2007, 87, 1999-2017 DOI: $10.1080 / 14786430701342164$.

(48) Reznitsky, A.; Klochikhin, A.; Permogorov, S.; Tenishev, L.; Lundin, W.; Usikov, A.; Schmidt, M.; Klingshirn, C. Localization of Excitons at Small In Clusters in Diluted InGaN Solid Solutions. phys. stat. sol. (c) 2003, 0, 280-283 DOI: 10.1002/pssc.200390043.

(49) Hammersley, S.; Watson-Parris, D.; Dawson, P.; Godfrey, M. J.; Badcock, T. J.; Kappers, M. J.; Mcaleese, C.; Oliver, R. A.; Humphreys, C. J. The consequences of high injected 
carrier densities on carrier localization and efficiency droop in $\mathrm{InGaN} / \mathrm{GaN}$ quantum well structures. J. Appl. Phys. 2012, 111, 083512 DOI: 10.1063/1.3703062.

(50) Chichibu, S. F.; Uedono, A.; Onuma, T.; Haskell, B. A.; Chakraborty, A.; Koyama, T.; Fini, P. T.; Keller, S.; DenBaars, S. P.; Speck, J. S.; et al. Origin of defect-insensitive emission probability in In-containing (Al,In,Ga)N alloy semiconductors. Nature Materials 2006, 5, 810 DOI: $10.1038 /$ nmat 1726.

(51) Liu, Q.; Lu, J.; Gao, Z.; Lai, L.; Qin, R.; Li, H.; Zhou, J.; Li, G. Electron localization and emission mechanism in wurtzite (Al, In, Ga)N alloys. physica status solidi (b) 2010, 247, 109114 DOI: $10.1002 / p s s b .200945258$.

(52) Lee, B.; Wang, L.-W. Band gap bowing and electron localization of $\mathrm{Ga}_{\mathrm{x}} \mathrm{In}_{1-\mathrm{x}} \mathrm{N}$. 2006, 100, 093717 DOI: $10.1063 / 1.2364450$.

(53) Wu, X.; Walter, E.; Rappe, A.; Car, R.; Selloni, A. Hybrid density functional calculations of the band gap of $\mathrm{Ga}_{\mathrm{x}} \mathrm{In}_{1-\mathrm{x}} \mathrm{N}$. Phys Rev B 2009, 80, 115201 DOI: 10.1103/PhysRevB.80.115201.

(54) Taylor, E.; Fang, F.; Oehler, F.; Edwards, P. R.; Kappers, M. J.; Lorenz, K.; Alves, E.; Mcaleese, C.; Humphreys, C. J.; Martin, R. W. Composition and luminescence studies of InGaN epilayers grown at different hydrogen flow rates. Semicond. Sci. Technol. 2013, 28, 065011 DOI: $10.1088 / 0268-1242 / 28 / 6 / 065011$.

(55) Rhode, S. L.; Fu, W. Y.; Moram, M. A.; Massabuau, F. C. P.; Kappers, M. J.; Mcaleese, C.; Oehler, F.; Humphreys, C. J.; Dusane, R. O.; Sahonta, S. L. Structure and strain relaxation effects of defects in InxGa1-xN epilayers. J. Appl. Phys. 2014, 116, 103513 DOI: 10.1063/1.4894688. 
(56) Lozano, J. G.; Nellist, P.; Guerrero-Lebrero, M.; Galindo, P. Effect Of The Eshelby-Stroh Twist In Dislocations In GaN Imaged End-On. Microscopy and Microanalysis 2012, 18, 13381339 DOI: $10.1017 / \mathrm{S} 1431927612008549$.

(57) Oliver, R. A.; Bennett, S. E.; Zhu, T.; Beesley, D. J.; Kappers, M. J.; Saxey, D. W.; Cerezo, A.; Humphreys, C. J. Microstructural origins of localization in $\mathrm{InGaN}$ quantum wells. Journal of Physics D: Applied Physics 2010, 43, 354003 DOI: 10.1088/0022$3727 / 43 / 35 / 354003$.

(58) Duff, A. I.; Lymperakis, L.; Neugebauer, J. Understanding and controlling indium incorporation and surface segregation on $\operatorname{In}_{x} \mathrm{Ga}_{1-\mathrm{x}} \mathrm{N}$ surfaces: $\mathrm{An}$ ab initio approach. Phys Rev $B$ 2014 DOI: 10.1103/PhysRevB.89.085307.

(59) Northrup, J. E.; Romano, L. T.; Neugebauer, J. Surface energetics, pit formation, and chemical ordering in InGaN alloys. Applied Physics Letters 1999, 74, 2319-2321 DOI: $10.1063 / 1.123837$.

(60) Hÿtch, M. J.; Snoeck, E.; Kilaas, R. Quantitative measurement of displacement and strain fields from HREM micrographs. Ultramicroscopy 1998, 74, 131-146 DOI: 10.1016/S03043991(98)00035-7.

(61) Cliff, G., Lorimer, G. W. The quantitative analysis of thin specimens. 1975, 103, 203207 DOI: 10.1111/j.1365-2818.1975.tb03895.x.

(62) McKenna, K. P. Electronic and Chemical Properties of a Surface-Terminated Screw Dislocation in MgO. J. Am. Chem. Soc. 2013, 135, 18859-18865 DOI: 10.1021/ja408342z. 
(63) Sugiyama, I.; Shibata, N.; Wang, Z.; Kobayashi, S.; Yamamoto, T.; Ikuhara, Y. Ferromagnetic dislocations in antiferromagnetic NiO. Nature Nanotechnology 2013, 8, 266-270 DOI: $10.1038 /$ nnano.2013.45.

(64) Stukowski, A. Visualization and analysis of atomistic simulation data with OVITO-the Open Visualization Tool. Modelling Simul. Mater. Sci. Eng. 2009, 18, 015012 DOI: 10.1088/0965-0393/18/1/015012. 